\title{
RNA Ensembles From Solvent Accessibility Data: Application to the SAM-I Riboswitch Aptamer Domain
}

\author{
Jingru Xie ${ }^{1}$ and Aaron T. Frank ${ }^{2,3^{*}}$
}

${ }^{1}$ Physics Department, University of Michigan, Ann Arbor, Michigan 48109, USA

${ }^{2}$ Biophysics Program, University of Michigan, Ann Arbor, Michigan 48109, USA

${ }^{3}$ Chemistry Department, University of Michigan, Ann Arbor, Michigan 48109, USA

*afrankz@umich.edu

\section{Benchmark RNAs}

\begin{tabular}{l|l}
\hline & PDB IDs \\
\hline \multirow{5}{*}{ Benchmark } & 1KKA 1L1W 1LDZ 1NC0 1OW9 1PJY 1R7W 1SCL 1UUU 1XHP 1YSV \\
& 1ZC5 2FDT 2JWV 2K66 2KOC 2L3E 2LBJ 2LBL 2LDT 2LHP 2LK3 2LP9 \\
& 2LPA 2LQZ 2LU0 2LUB 2LUN 2LV0 2M12 2M21 2M22 2M24 2M4W 2M5U \\
& 2M8K 2MEQ 2MFD 2MHI 2MNC 2N2O 2N2P 2QH2 2QH4 2Y95 \\
\hline
\end{tabular}

Table S1. PDB IDs of the 45 RNAs used to benchmark SASA-based BME reweighting. 

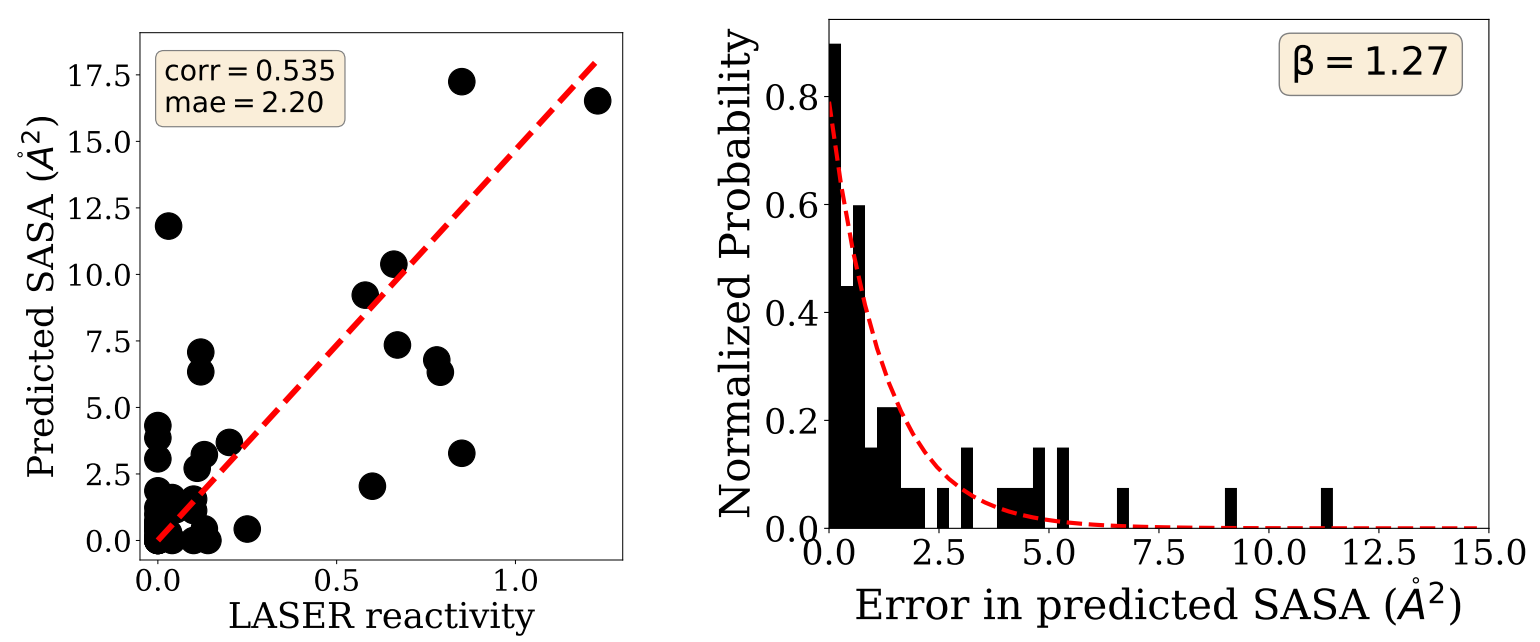

Figure S1. Linear Fit of C8-SASA to LASER reactivity. Left: the linear fit of C8-SASA to LASER reactivity. Right: exponential distribution of absolute error of the linear fit

\section{Adding noise to target SASA data.}

As a more stringent test of the power of C8-SASA in ensemble reweighting, we carried out tests with target SASA data that included random noise. By adding noise to target SASA, we attempted to mimic SASA data that would be derived from experimental observables which contain systematic or statistical error at various levels and exhibit strong but imperfect correlation with SASA. We sampled random white noise from an exponential distribution with mean ranging from 0.3 to $4.8 \AA^{2}$ with a random sign, and added it on to target SASA value for each atom. Note that the mean of error of $1.27 \AA^{2}$ corresponded to the SASA data derived from LASER experiments for SAM riboswitch. To mimic LASER-derived C8-SASA, we added random white noise to computed C8-SASA with mean of $1.27 \AA^{2}$. The value of $1.27 \AA^{2}$ was estimated from the error of LASER derived C8-SASA. We made a linear fit on computed SASA to LASER reactivity, and the absolute value of error of the fit was best estimated by an exponential distribution with scale parameter $\beta=1.27$ (Figure S1). The resulting linear fit had a correlation score of 0.535 (and the mean absolute error was $2.69 \AA^{2}$ ).

At a given noise-level, we carried out BME reweighting trials on 500 noisy data sets. We did this to mitigate bias in our analysis from a single noisy data set. Preliminary analysis revealed that mean weights for individual conformers within a given conformational pool converged over 500 trials (See Figure S2). 


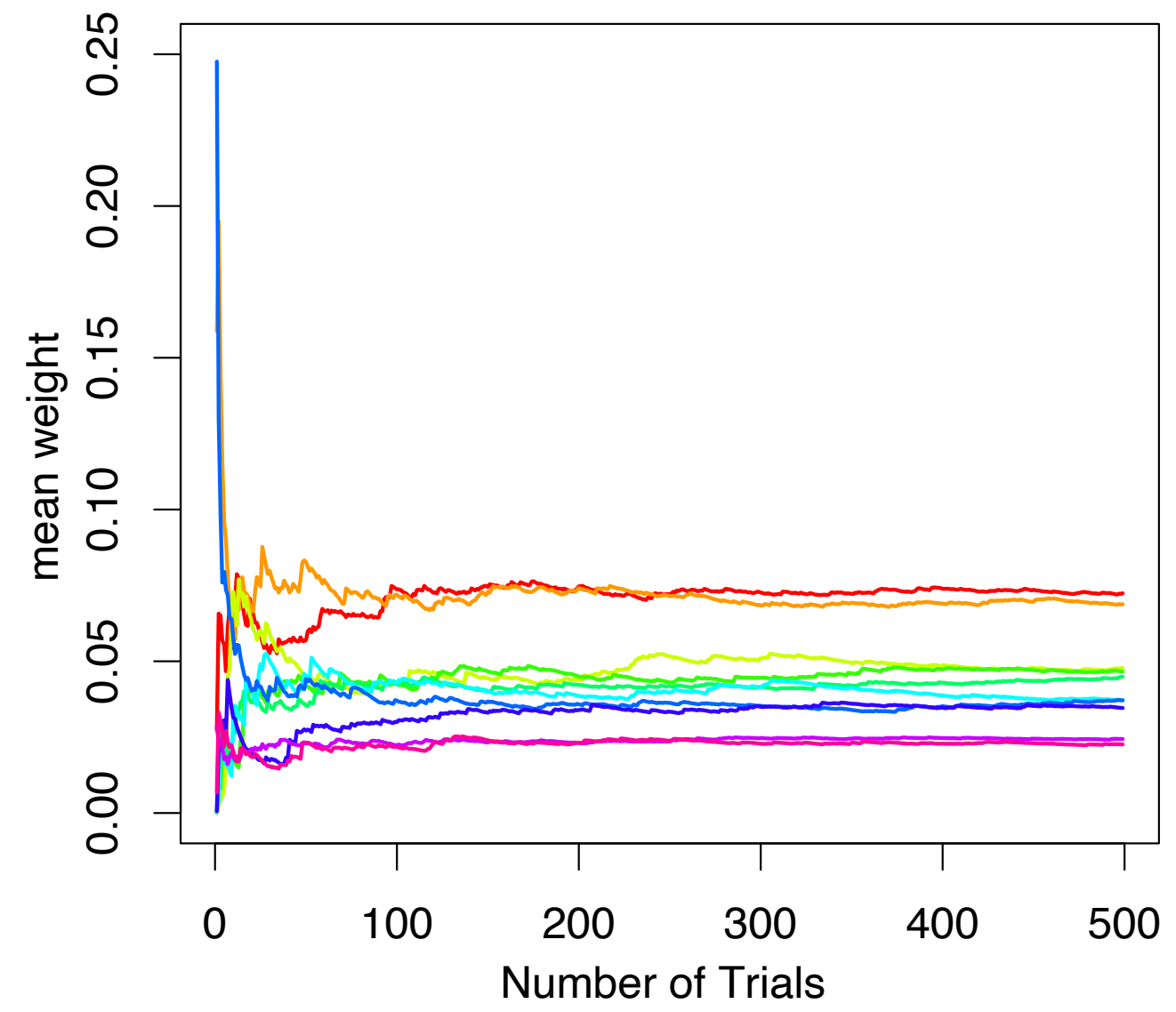

Figure S2. This figure illustrates how the cumulative moving average of BME weights changes with increased number of trials when performing benchmarking with added random noise. Shown in different colors are mean weights of the 10 highest weighted conformers in the 2LU0 benchmark set with target ensemble width of $5.0 \AA$ 


\section{Difference atomic density maps for benchmark RNAs.}

To visually inspect individual ensembles, we calculated the difference between the atomic density maps (diff maps) of the target $(T)$ and the decoy $(D)$ ensembles and compared them to the diff maps of the target $(T)$ and the reweighted $(R)$ ensembles. Analysis of the difference maps indicated that in contrast to decoy ensembles, the ensembles reweighted with noise-free SASA generally exhibited small residual densities relative to the target densities, confirming that conformational distribution in these ensembles closely mirrored the target. Ensembles that were reweighted with SASA data that contained simulated noise exhibited larger residual densities relative to target ensembles than their corresponding noise-free counterparts, but smaller residual densities than decoy ensembles. These results provide a visual confirmation that, indeed, the C8-SASA can be used to reweight the decoy ensembles toward the target from which the SASA was calculated. We note, however, that when noise is added to the target C8-SASA data, the reweighted ensembles do not perfectly recapitulate the target ensemble. The difference atomic density maps as well as PyMOL script to visualize them can be accessed via https://doi.org/10.5281/zenodo.4392866. This script define a function that can be used to render diff PyMOL as fofc volume maps, in which green and red color indicate $-/+3$ sigma contours. 

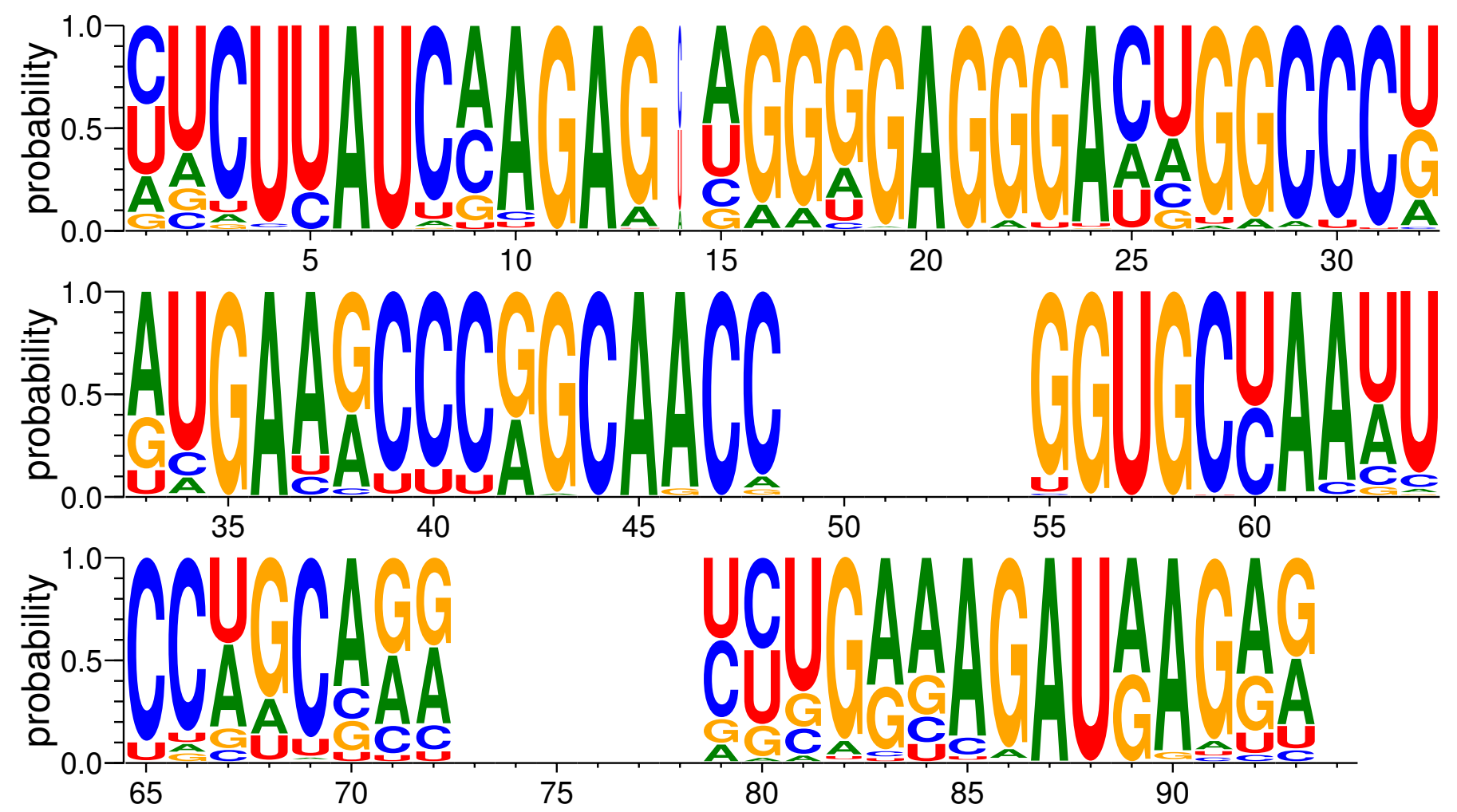

Figure S3. Conservation map for SAM-I riboswitch. 


\section{SAM Folding Simulations}

Gō-like all-atom folding simulations of the SAM-aptamer were carried out using SMOG-2. ${ }^{1}$ The Gō-like structured-based force field used for these simulations were set up based on the apo structure of the SAM aptamer (PDBID: $3 \mathrm{IQP}^{2}$ ). The RNA was first unfolded by carrying out 100000 steps of Langevin dynamics with the temperature set to $500 \mathrm{~K}$. To trigger refolding, the unfolded structures were then thermally quenched by running Langevin dynamics with the temperature set to $50 \mathrm{~K}^{3}$. A set of 100 independent such refolding simulations were carried out over 10,000,000 simulation steps. For all simulations, the Langevin friction $(\gamma)$ was set to 1.0 ps $^{-1}$, the Coulomb and L-J cutoff distance were set to $15 \AA$, and the integration time-step was set to 0.0005 ps. The simulations were carried out using OpenMM ${ }^{4}$. Productive folding occurred in 57 of the 100 trajectories. The results presented in the manuscript were based on the analysis of these 57 folding trajectories. For each of the 57 refolding simulations, the RMSD relative to the apo structure of the SAM aptamer was determined along the trajectory. From the RMSD timeseries data the folding times were determine as the first passage time for the RMSD to be less $2.0 \AA$. To determine the contact times for A24-A85 and U64-A85 along the 57 trajectories, we computed the distance timeseries between atoms N6 on A24 and N3 on A85 and N3 U64 and $\mathrm{N} 1 \mathrm{~A} 85$, respectively, and then calculated the first passage time for the distances to be less than 3.5 A. These are the folding and contact times presented in the main manuscript. The scripts and input files used to carry out the folding simulations and subsequent analysis is available at https://doi.org/10.5281/zenodo.4392866. 


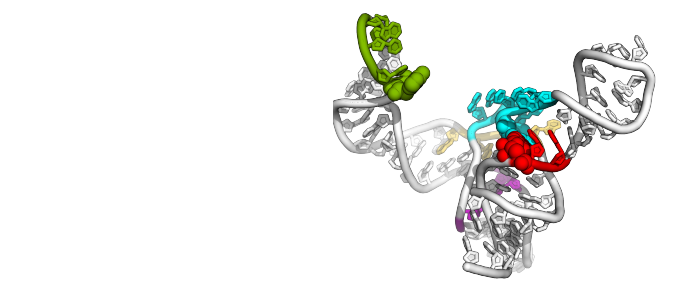

Model: Folding Replica 1

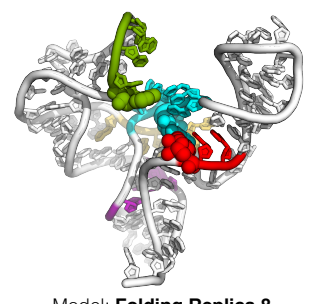

Model: Folding Replica 8
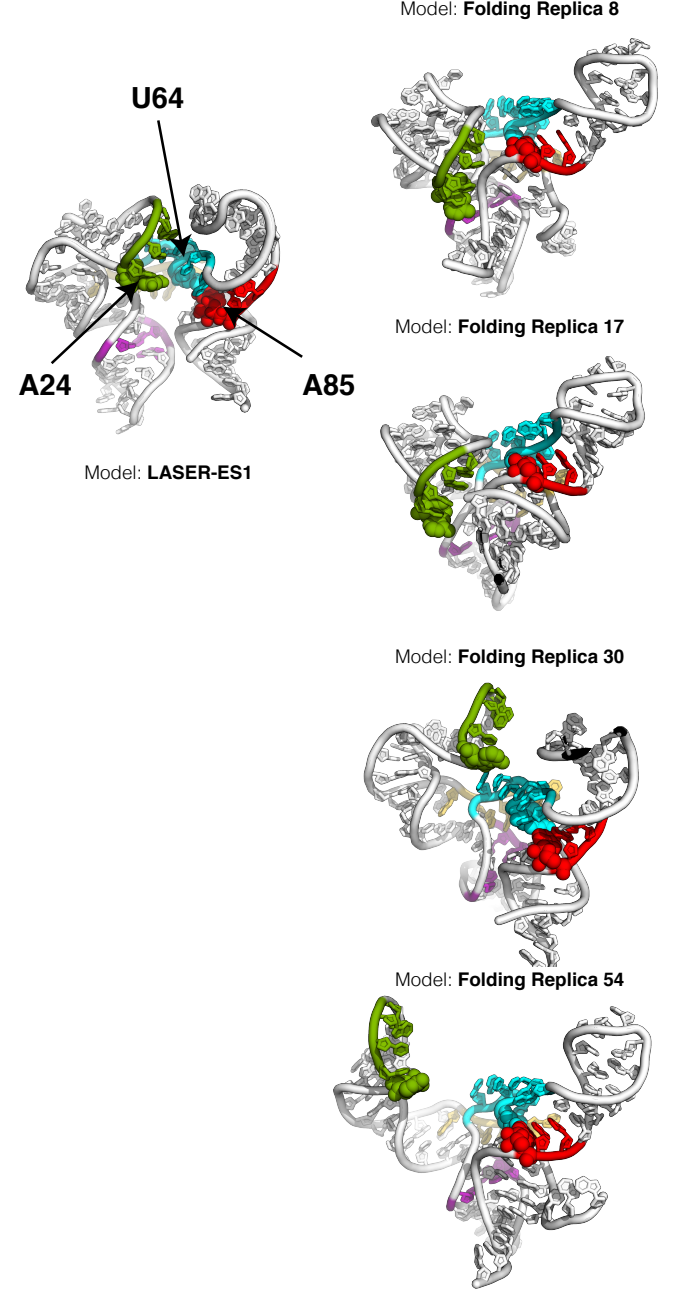

Model: Folding Replica 88
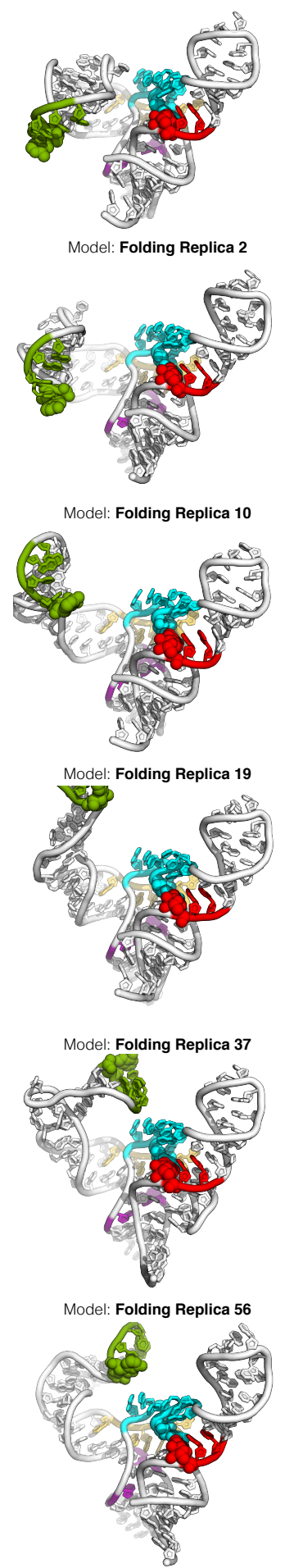

Model: Folding Replica 95
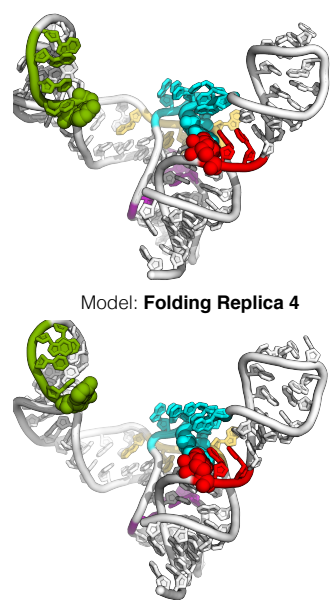

Model: Folding Replica 12
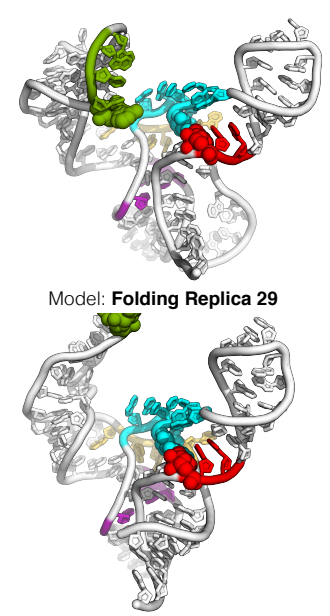

Model: Folding Replica 43

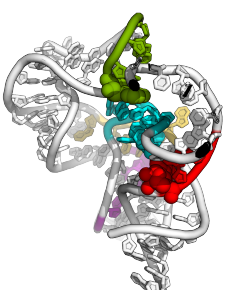

Model: Folding Replica 77

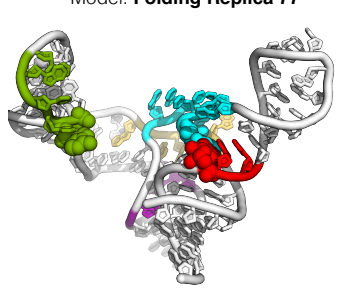

Model: Folding Replica 96

Figure S4. Shown is a comparison between ES1 in the LASER -SAM ensemble and SAM-aptamer conformations harvested from 18 of 100 Gō-like all-atom folding that like ES1 were semi-folded but lacked the long range A24-A85 contact found in the apo crystal structure. 


\begin{tabular}{lccc}
\hline Structure & Apo RMSD $(\AA)$ & A24-A85 Distance $(\AA)$ & A24-A85 Distance $(\AA)$ \\
\hline LASER -SAM: ES1 & 7.0 & 12.0 & 2.8 \\
Folding Replica: 1 & 7.9 & 24.0 & 3.1 \\
Folding Replica: 2 & 7.9 & 33.6 & 2.9 \\
Folding Replica: 4 & 7.9 & 25.7 & 3.4 \\
Folding Replica: 8 & 8.0 & 10.7 & 3.1 \\
Folding Replica: 10 & 8.0 & 30.7 & 2.7 \\
Folding Replica: 12 & 7.9 & 27.0 & 2.6 \\
Folding Replica: 17 & 7.9 & 19.1 & 3.0 \\
Folding Replica: 19 & 8.0 & 25.5 & 2.9 \\
Folding Replica: 29 & 8.0 & 14.8 & 3.2 \\
Folding Replica: 30 & 7.8 & 21.4 & 3.2 \\
Folding Replica: 37 & 7.8 & 35.9 & 3.1 \\
Folding Replica: 43 & 7.9 & 34.5 & 3.1 \\
Folding Replica: 54 & 7.7 & 18.2 & 2.9 \\
Folding Replica: 56 & 8.0 & 32.7 & 2.9 \\
Folding Replica: 77 & 7.9 & 14.9 & 3.1 \\
Folding Replica: 88 & 8.0 & 23.3 & 2.8 \\
Folding Replica: 95 & 7.9 & 23.4 & 3.1 \\
Folding Replica: 96 & 7.9 & 26.1 & 2.9 \\
\hline
\end{tabular}

Table S2. Listed for each is the RMSD relative to the apo crystal structure and the distance between N6 on A24 and N3 on A85 and N3 U64 and N1 A85, respectively for the ES1 structure in the -SAM ensemble and the 18 ES1 conformers harvested from the Gō-like all-atom folding trajectories (see Figure S4). 


\section{Impact of Sampling}

To assess the impact of sampling on the -SAM ensemble that emerges from the LASER/SASA BME reweighting, we generated a new conformational pool containing the original KGSrna structures (described in the main manuscript) and structures harvested from the SMOG folding trajectories (see above). In total 20 structures were harvested from the final half of each of the 100 refolding trajectories, resulting in a total of 2000 structures. Discarded from the set 2000 structures were those with RMSDs greater than $10 \AA$ relative to the apo SAM crystal structure. This filtering was carried out to remove unfolded structures. The 1268 structures that remained after filtering were added to the KGSrna structures and SASA-based reweighting was again carried out using BME. The comparison between the four highest weighted structures are shown in Figure S5.

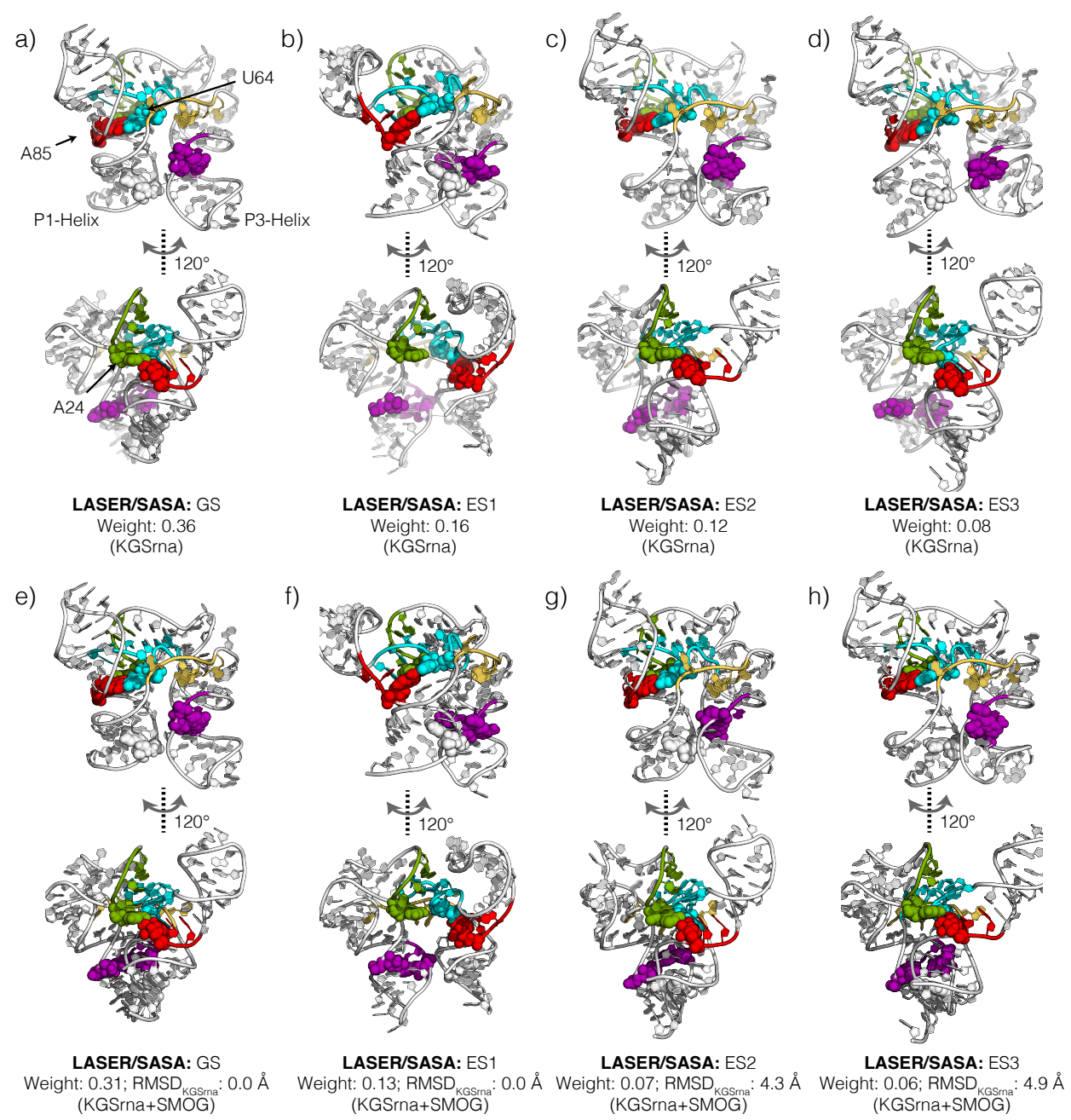

Figure S5. Comparison between four highest weighted conformers when reweighting the KGSrna (a-d) and KGSrna+SMOG (e-h) pools. The two highest weighted structures, GS and $E S 1$, in the KGSrna SASA BME-reweighted ensemble ( $a$ and $b$ ) were are identical to the first two weighted structures in the the KGSrna+SMOG SASA BME-reweighted ensemble (e and f). Though not identical, ES2 and ES3 in both ensemble resemble each other; their RMSDs 4.3 and $4.9 \AA$, respectively. As indicated, the KGSrna+SMOG weights were slightly lower. 


\section{Cavity mapping and docking experiments}

a)

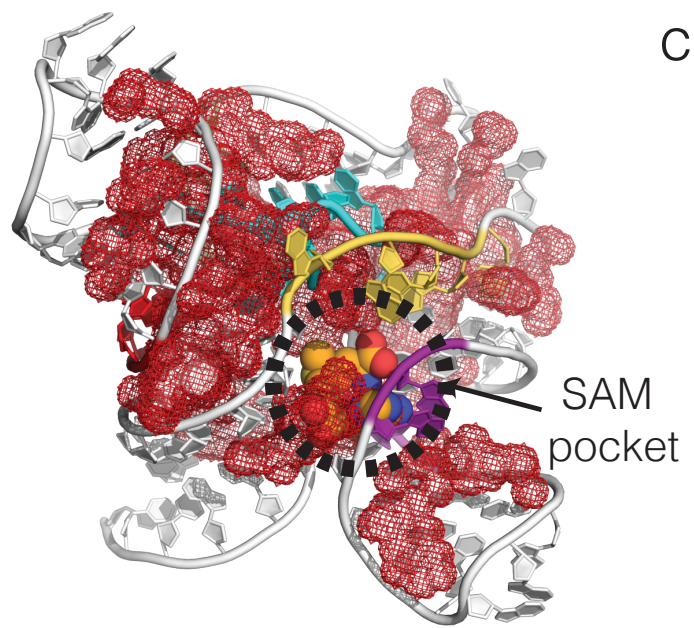

GS: Weight: 0.36

(Apo RMSD: $1.5 \AA$ )

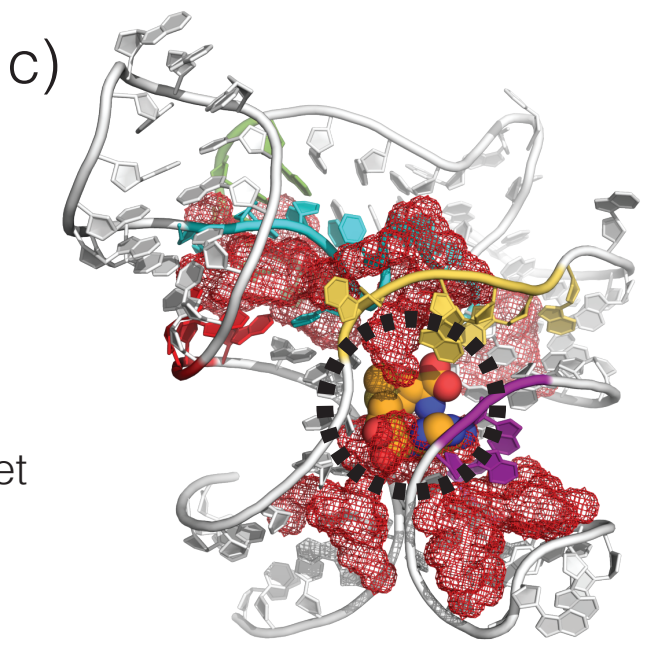

ES2: Weight: 0.12

(Apo RMSD: $2.3 \AA$ ) b)

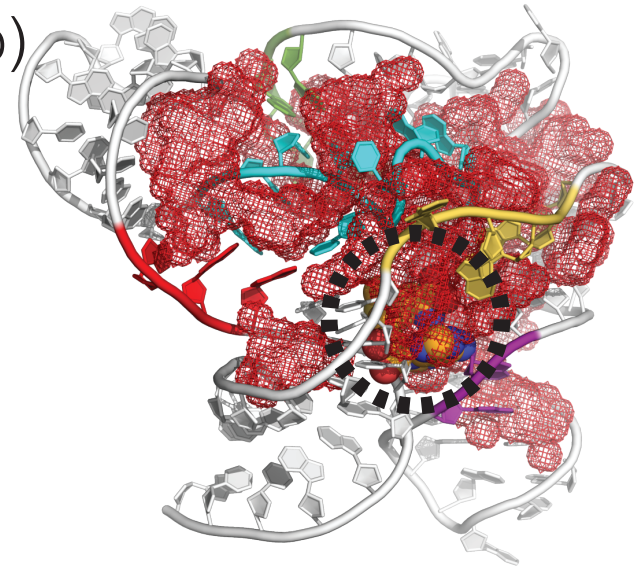

ES1: Weight: 0.16 (Apo RMSD: $7.0 \AA$ )

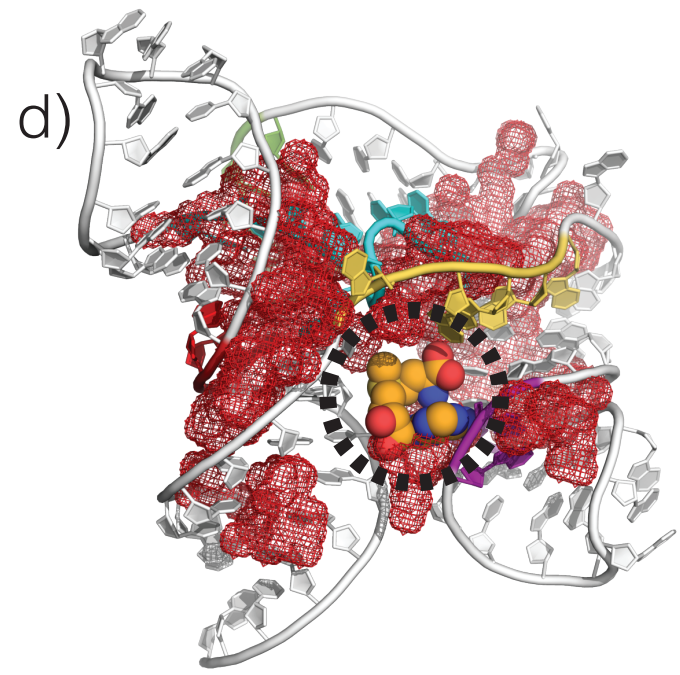

ES3: Weight: 0.08 (Apo RMSD: $1.41 \AA$ )

Figure S6. Cavity detected in the four highest weighted conformers in the -SAM ensemble of the SAM-I aptamer domain.

For each of the four highest weighted conformers in the -SAM ensemble, we carried out cavity mapping to identify sites on the surface that might facilitate interactions with smallmolecule ligands (Figure S6). To achieve this, we utilized the two-sphere cavity mapping method implemented in the rbcavity program within the rDock modeling suite 5 . For cavity mapping, we set the maximum number of cavities to 10 and the minimum cavity volume to $50 \AA^{2}$. The resulting cavities were visualized in PyMOL (version 2.3.4). Next, we carried out a small-scale in silico screening by docking 500 small, drug-like molecules into the cavities identified using rbcavity. These 500 small molecules corresponded to a small library of drug-like compounds obtained 
from the ZINC library ${ }^{6}$. To identify the most conformationally selective compounds in the library, we first computed the selectivity index, $\gamma_{i, j}$, which we defined as

$$
\begin{aligned}
\gamma_{i, j} & =\frac{\Delta G_{i, j}}{\left\langle\Delta G_{i, j}\right\rangle} \\
\gamma_{i} & =\max _{j} \gamma_{i, j}
\end{aligned}
$$

Here $i$ runs over the compounds in the library, $j$ runs over the conformers (docking receptors), $\Delta G_{i, j}$ is the docking score for compound $i$ docked onto conformer $j$, and $\left\langle\Delta G_{i, j}\right\rangle$ is the average docking score for compound $i$ across all $j$ conformers. For each compound, we assign a selectivity $\left(\gamma_{i}\right)$ as the maximum of the set of selectivity indices $\left\{\gamma_{i, j}\right\}$. As defined, compounds with high $\gamma_{i}$ correspond to those that have a docking score $\left(\Delta G_{i, j}\right)$ on a given conformer that is significantly more favorable (negative) than the average docking score $\left(\left\langle\Delta G_{i, j}\right\rangle\right)$; such a compound was identified as being a "conformationally selective" compound. The scripts and input files used to carry out the docking calculations and subsequent analysis is available at https://doi.org/10.5281/zenodo.4392866.

\section{References}

1. Noel, J. K. et al. SMOG 2: a versatile software package for generating structure-based models. PLoS computational biology 12, e1004794 (2016).

2. Stoddard, C. D. et al. Free state conformational sampling of the SAM-I riboswitch aptamer domain. Struct. 18, 787-797 (2010).

3. Whitford, P. C. et al. Nonlocal helix formation is key to understanding S-adenosylmethionine-1 riboswitch function. Biophys. journal 96, L7-L9 (2009).

4. Eastman, P. et al. OpenMM 4: a reusable, extensible, hardware independent library for high performance molecular simulation. J. chemical theory computation 9, 461-469 (2013).

5. Ruiz-Carmona, S. et al. rDock: a fast, versatile and open source program for docking ligands to proteins and nucleic acids. PLoS Comput. Biol 10, e1003571 (2014).

6. Sterling, T. \& Irwin, J. J. ZINC 15-ligand discovery for everyone. J. chemical information modeling 55, 2324-2337 (2015). 